\title{
DISEÑO DE PÁGINAS WEB EDUCATIVAS PARA TELÉFONOS MÓVILES
}

\author{
Francisco Brazuelo Grund; brazuelo3@hotmail.com \\ IES Faro de Maspalomas, España \\ Maria Luz Cacheiro González; mlcacheiro@edu.uned.es \\ UNED, España
}

\section{RESUMEN}

Presentamos a continuación un trabajo de investigación acerca del diseño de páginas web para teléfonos móviles en el ámbito educativo. En el marco teórico, estudiaremos la situación actual de la telefonía móvil como recurso educativo. A continuación estableceremos un marco de actuación metodológica basado en herramientas de la Web 2.0, llegando finalmente a la creación de la web móvil Diseño de Páginas Web en Contextos Educativos, perteneciente al programa de doctorado MODELTIC de la UNED.

PALABRAS CLAVE: teléfono móvil y educación, mobile learning, Web 2.0, diseño web

\section{ABSTRACT}

We are presenting a research paper about designing web pages for mobile phones in an educational context. In the theoretical framework, we will study the current situation of mobile telephony as an educational resource. Then we will establish a framework of methodological approach based on the tools of the Web 2.0, finally arriving to the creation of mobile Web 'Diseño de Páginas Web en Contextos Educativos,' as part of the doctoral program at UNED MODELTIC.

KEYWORDS: mobile phone and education, mobile learning, Web 2.0, Web Design

\section{INTRODUCCIÓN}

El teléfono móvil se ha convertido en el medio de comunicación más extendido del mundo superando a la prensa escrita, la televisión e Internet (Ahonen y Moore 2008). Actualmente se estima que hay más de 4.000 millones de estos dispositivos y las cifras siguen en aumento. En el caso español, según la Comisión del Mercado de las Telecomunicaciones en su informe de enero del 2009, la tasa de penetración de la telefonía móvil supera el 107\% de la población total, es decir, que tenemos más teléfonos móviles que habitantes, concretamente, más de 51 millones.

Por otro lado, la telefonía móvil es un mercado en continua evolución y renovación tecnológica. Un teléfono móvil ya no se limita a permitir realizar llamadas sino que está ampliando, cada vez 
más, sus posibilidades comunicativas, multimedia y ofimáticas: podemos enviar SMS y MMS, hacer fotos, grabar audio y vídeo, almacenar datos, música y películas, orientarnos por GPS, crear y editar documentos y hojas de cálculo, conectarnos vía Wi-Fi y, por supuesto, navegar por Internet. Además la nueva generación de terminales táctiles con sus nuevos sistemas operativos y la posibilidad de instalar aplicaciones de terceros, están convirtiendo al teléfono móvil en un pequeño ordenador de bolsillo. Todas estas posibilidades unidas a su relativo bajo coste, ubicuidad, facilidad de uso y portabilidad, justifican la, anteriormente comentada, expansión mundial.

De las posibilidades comunicativas anteriormente citadas, la que está contando con una con mayor perspectiva de rápido crecimiento es la navegación por Internet. El Informe Horizon (2008), que marca las tendencias sobre las tecnologías emergentes, prevé la implantación de la banda ancha móvil en un plazo de dos o tres años y en una década el teléfono móvil se convertirá en el principal medio de acceso a Internet.

Uno de los segmentos sociales que más está reflejando este impacto de la telefonía móvil son los jóvenes. Ya sabíamos que el $100 \%$ de los jóvenes disponían de teléfono móvil. Pero lo que desconocíamos hasta ahora es que, además, cada vez son más los que están accediendo a Internet a través del móvil. Según los últimos datos publicados por el "3er Observatorio de Tendencias" encargado por la marca Nokia a la consultora de investigación social y de mercado Conecta (julio 2009), el porcentaje de personas comprendidas entre 15 y 35 que se conectan a Internet desde un móvil se ha triplicado en un año pasando de un $8 \%$ al actual $24 \%$ con una media de 2,6 horas semanales.

A pesar de estos datos, nuestra realidad educativa muestra una perspectiva completamente distinta y contradictoria, contrapuesta totalmente a la realidad social. Y se manifiesta en la gran resistencia a la integración de los teléfonos móviles como una herramienta educativa más en el contexto escolar. Creemos que tres son las grandes barreras para esta integración educativa: en primer lugar las administraciones educativas, en todos los niveles, han dictado directrices para la restricción o total prohibición de los móviles en los centros educativos. En segundo lugar, el alumnado no tiene conciencia de que el móvil pueda ser utilizado con fines educativos, lo consideran solo como un medio lúdico. En tercer lugar, el profesorado, en general, desconoce la potencialidad educativa de estos dispositivos que contemplan como un elemento disruptivo o de intromisión en el aula. Sin embargo, no podemos dar la espalda a la realidad cotidiana: el teléfono móvil constituye un elemento integrante de nuestra vida cotidiana y diríamos que hasta imprescindible en la de nuestro alumnado.

Con este trabajo pretendemos ofrecer una muestra de cómo utilizar el teléfono móvil como una herramienta educativa mediante una ejemplificación de diseño y elaboración de una página web móvil a partir de la adaptación de la Guía didáctica del curso Diseño de páginas web en contextos educativos (Cacheiro 2008).

\section{FUNDAMENTACIÓN TEÓRICA}

Los avances tecnológicos abren las puertas a nuevas formas y oportunidades de acceso al aprendizaje. Por ello, de forma paralela al desarrollo de las tecnologías móviles en general, y la telefonía móvil en particular, ha surgido una corriente, relativamente reciente (aproximadamente una década) de investigaciones interesadas en estudiar las implicaciones y las aplicaciones de dispositivos electrónicos móviles en el campo educativo: el Mobile Learning.

\subsection{Mobile Learning}




\subsubsection{Hacia una definición de Mobile Learning}

La terminología más aceptada para referirnos a los estudios de investigación que relacionan movilidad, aprendizaje y dispositivos electrónicos móviles es Mobile Learning. Pero no es la única. También se utilizan términos abreviados como m-Learning y MLearning. En su forma castellanizada se utiliza el término "aprendizaje móvil". Desde aquí y ahora nosotros utilizaremos solo la primera, es decir, Mobile Learning (ML).

El ML es un campo de investigación relativamente reciente (aproximadamente una década) y por tanto en evolución en el cual no hay unanimidad sobre qué es el ML (Caudill 2007). Winters (2006) señalaba tres tendencias en relación a la definición de ML: tecnocéntrica; evolutiva a partir del e-Learning; y centrada en el estudiante y su contexto.

En los inicios del ML, los investigadores habían definido el ML como la aplicación de los dispositivos electrónicos móviles para fines educativos (tales como reproductores de MP3, una PDA, un smarthphone, un iPod, Netbook, Tablet PC o un teléfono móvil). Por tanto tecnología móvil y aprendizaje aparecían indisolublemente unidos (Traxler 2005; Parsons y Ryu, 2006).

Otros, sin embargo, consideraron el ML como una evolución del e-Learning e incorporaron el valor de la ubicuidad total del aprendizaje, es decir, poder aprender en cualquier momento y cualquier lugar (Quinn 2000). El e-Learning permite una gran flexibilidad espacial y temporal pero dependiente de la necesidad física de un ordenador y una conexión a Internet. Esto no sucede con el ML. No importa cuando sea cuando o donde estemos. Siempre podremos acceder a los contenidos de aprendizaje. Esto potencia el concepto de flexibilidad tradicionalmente atribuido al e-Learning (Georgiev, Georgieva y Trajovski, 2006).

Sin embargo una tercera tendencia considera el ML no como una evolución del e-Learning sino como una nueva modalidad de enseñanza a distancia y enfoca la definición hacia el estudiante en dos aspectos: su movilidad y su contexto de aprendizaje. Este concepto de ML como aprendizaje ubicuo ligado a la movilidad y el contexto de aprendizaje del alumno facilitado por las tecnologías móviles, ha servido para construir las bases para la primera teoría acerca del ML.

\subsubsection{Hacia una teoría del Mobile Learning}

La teoría del ML propuesta tiene dos conceptos centrales que giran en torno al estudiante: movilidad y contexto de aprendizaje. Consideran que lo importante, para empezar, no es la tecnología, sino el concepto de movilidad. Para Sharples, Taylor y Vacuola (2007) hay tres implicaciones del concepto "mobile" en la terminología de Mobile Leaning:

1.Movilidad física. Las personas se encuentran en continuo movimiento y aprenden a diario en diversas situaciones cotidianas. Por tanto no podemos obviar el contexto situacional en el aprendizaje.

2.Movilidad tecnológica. Disponemos de tecnologías móviles que podemos llevar consigo en todo momento y lugar de una forma fácil y cómoda.

3.Movilidad social. El aprendizaje puede tener un componente social. Podemos aprender de y con los demás en diversos entornos, ya sea un aula o en un entorno familiar o laboral.

Por tanto, es el estudiante lo que mueve y con él cualquier tecnología móvil que lleve consigo. Debe considerarse a ésta no como un fin en $\mathrm{ML}$, sino solo un medio facilitador de oportunidades de aprendizaje, especialmente cuando existe movimiento físico. $Y$ si nos movemos, cambia el contexto de aprendizaje. 
El contexto es un constructo central para el ML. Evidentemente, el aprendizaje no ocurre en el vacío sino que se construye en el espacio y el tiempo y a través, generalmente, de interacciones personales. El problema surge cuando trascendemos del tradicional espacio el aula y una intermediación docente. La construcción de significados a través del aprendizaje se da entonces en cualquier momento y lugar. Gracias a la era digital y al nacimiento del ciberespacio, hemos roto las barreras del aula tradicional y el acceso a la información se ha universalizado. Ahora, además, contamos con tecnologías que pueden acompañarnos a través de nuestro "nomadismo" diario y pueden facilitarnos el aprendizaje de forma totalmente ubicua, pudiendo hacer confluir, incluso, contextos de aprendizaje real y virtual, cubriendo demandas de aprendizaje adaptadas al estudiante.

Investigar acerca del ML se convierte, por tanto, en estudiar acerca de la movilidad de los estudiantes y sus oportunidades de aprendizaje en diversos contextos, aprendizaje que puede verse incrementado gracias a la tecnología. Son muchas las experiencias, proyectos y pruebas piloto las que se han realizado hasta ahora con las tecnologías móviles en diversos contextos situacionales que han demostrado un considerable potencial pedagógico del ML.

\subsubsection{Ventajes del Mobile Learning}

Como hemos visto, el ML permite extender el aprendizaje más allá de las limitaciones físicas de un aula o de un ordenador de sobremesa. Se han señalado algunas ventajas del ML (Wagner 2005; Kukulska-Hume 2007) como:

- Permite el aprendizaje en cualquier momento y lugar.

- Puede mejorar la interacción didáctica forma síncrona y asíncrona.

- Potencia el aprendizaje centrado en el alumno

- Enriquecimiento multimedia del aprendizaje

- Permite la personalización del aprendizaje

- Favorece la comunicación entre el alumnado y las instituciones educativas.

- Favorece el aprendizaje colaborativo.

\subsection{Web 2.0 y Mobile Web 2.0}

\subsubsection{Web 2.0}

Podemos considerar la Web 2.0 como el paso evolutivo de la tecnología y el lenguaje web hacia la dinámica y la interacción social en la red en contraste con la "quietud" y el "silencio" que representaba la Web 1.0. La Web 2.0 es comunicación, un continuo fluir conversacional, además de colaborativa y participativa.

El término fue propuesto por O’Reilly (2006), fundador de O’Reilly Media y acérrimo defensor del software libre, para significar un cambio, un "algo nuevo está pasando" en el mundo de la Web entorno al 2004, pasado el momento del estallido de la burbuja tecnológica de principios del 2000.

En la Web 1.0 el usuario es un mero receptor de contenidos creados en una deteminado site por un administrador. En la Web 2.0, el usuario es el protagonista y tiene un papel muy activo en la creación, edición, actualización y compartición de contenidos. La Web 1.0 es unidireccional, la Web 2.0 es bidireccional. 
Esto ha dado lugar a todo un mundo de aplicaciones, utilidades y servicios comunicativos e interactivos que fomentan la colaboración social e intercambio de información tales como los blog o bitácoras, vblogs o videoblogs, wikis, redes sociales (como Facebook o Tuenti), redes de sindicación RSS, mashup, tag clouds o nubes de etiquetas, y un largo etcétera. Y no nos olvidemos de la folksonomía.

Nuestros jóvenes, especialmente, no son ajenos a estos cambios, más bien al contrario, son sus auténticos protagonistas. Fuera del aula invierten mucho tiempo en estar conectados a redes sociales y colaborativas. Es la Generación de Nativos Digitales nacida en los noventa que han crecido rodeadas de tecnologías hipermediales y multimedia. A esta generación le gusta aprender con y de los demás, compartir información y son grandes creadores de contenidos (Kolb 2008). Y, como ya vimos anteriormente, son cada vez más los que acceden al mundo de la sociedad virtual a través del teléfono móvil.

\subsubsection{Mobile Web 2.0}

¿Qué es Mobile Web 2.0? Mobile Web 2.0 es combinar lo mejor de dos mundos, la Web 2.0 y los dispositivos móviles (Nations 2009). El Mobile Web 2.0 ha evolucionado del Mobile Learning y de la Web 2.0, en concreto de la adaptación de la Web 2.0 a las tecnologías móviles (Coppola 2008). El Mobile Web 2.0 permite el acceso a numerosos recursos de la Web 2.0 adaptados a las características de los dispositivos móviles como moblogs (blog para móviles), mowikis (wikis para móviles), podcast, mashups, m-Voip (telefonía con tecnología Voip para móviles), redes sociales, etc.

Hasta ahora habíamos asistido al intento de adaptar la tecnología WAP a los teléfonos móviles. Pero la navegación en Internet por este sistema se mostraba (y se muestra aún en la mayoría de los teléfonos móviles) incómoda para el usuario debido a razones de operabilidad, incompatibilidad, accesibilidad y usabilidad tales como: lentitud de navegación; webs que no se parecían nada a las que los usuarios estaban acostumbrados a ver cuando navegaban en un ordenador convencional; incomodidad a la hora de navegar por las páginas web; pantallas muy reducidas para una correcta visualización de las web; introducción de datos e información incómoda debido a los miniteclados de los móviles que además solo permitían maniobrar con una sola mano; tarifas de conexión caras; carecen de un sistema de navegación por ventanas, lo que permite visualizar una página cada vez y únicamente de forma lineal, entre otras razones (Banerjee y Dholakia 2008).

Sin embargo, nos encontramos en el momento para la segunda oportunidad en el desarrollo de la Web Móvill. La extensión de las redes WiFi para móviles $3 \mathrm{G}$ facilitan el acceso a Internet desde prácticamente cualquier lugar; existen herramientas Web 2.0 que facilitan enormemente la creación de blogs y web para móviles y lo más importante es que ahora podemos acceder a servicios tan populares como el correo electrónico, los programas de chat y mensajería electrónica y el servicio estrella de Internet: las redes sociales, lo que ha triplicado, en un solo año, el acceso a Internet a través del móvil (Durán 2009).

\subsection{Diseño web para dispositivos móviles}

Sin embargo no es lo mismo diseñar una web para un ordenador de sobremesa que para un teléfono móvil. La diferencia más evidente, y determinante, es el tamaño de la pantalla y esta diferencia condiciona no solo el diseño sino la propia selección del contenido: no todo cabe en la pantalla de un móvil. Por tanto diseño y contenido deben de estar adaptados al contexto móvil. 
Y es que la clave principal cuando se trata de diseñar sitios web para dispositivos móviles es la simplicidad. Y se busca simplicidad para alcanzar una de las claves de éxito en una web: rapidez de carga. Otra razón es, ya lo hemos comentado, el tamaño de la pantalla. La web no puede estar muy saturada de elementos ya que, aparte de no poder visualizarse adecuadamente, ralentizaría de igual modo la conexión, hecho habitual al navegar por un dispositivo móvil. Por tanto se debe de seleccionar lo realmente importante y descartar lo innecesario ofreciendo al usuario opciones simples de acceso a los contenidos (Snell 2009).

En el siguiente cuadro (Villa 2007) podemos ver un esquema paso a paso de qué se debe de tener en cuenta a la hora de diseña una web móvil (Figura 1).

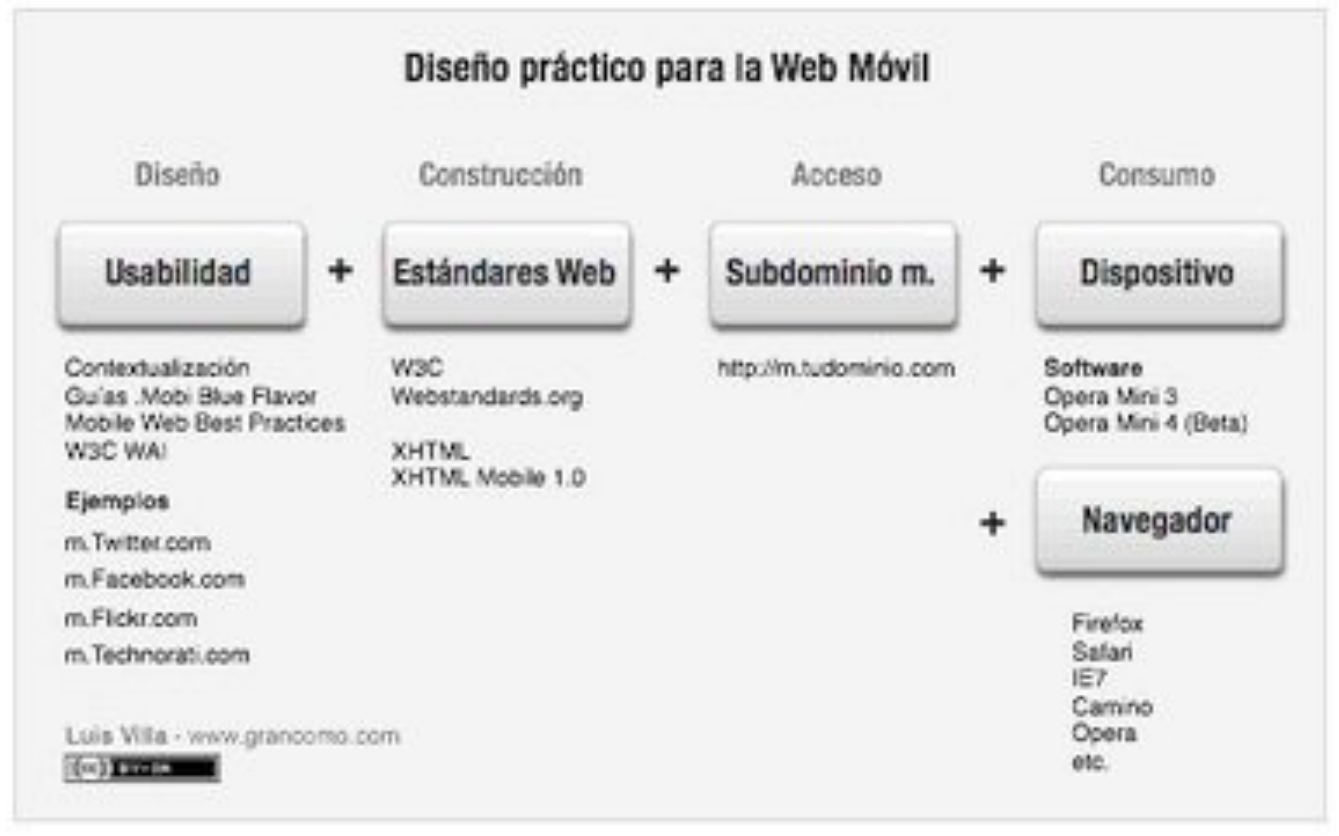

Figura 1. Diseño práctico para web móvil

\subsubsection{Herramientas web 2.0 para el diseño de web móviles}

Una de las grandes ventajas de Web 2.0 es que no hay que tener conocimientos informáticos para crear, editar o compartir contenidos en la red. La Web 2.0 provee de múltiples y variadas herramientas y el diseño de web móviles no escapa a esta ventaja. De hecho crear web móviles es relativamente sencillo, rápido y gratuito. $\mathrm{Y}$ todo esto es muy importe en contextos educativos: llegará el día (y será pronto) en el que la elaboración de moblogs, webquest, mWikis o web móviles formarán parte de las tareas educativas que realizaremos para o con nuestros alumnos. Entre las 10 mejores herramientas Web 2.0 para la elaborar web para dispositivos móviles tenemos: WINKSITE (http://winksite.com); ZINADOO (http://www.zinadoo.com); UBIK (www.ubik.com); DOTMOBI (http://site.mobi/); JAGANGO (http://www.jag.ag); MOBISITEGALORE (http://www.mobisitegalore.com); FREEMOBIZ (http://www.freemobiz.com); MOBILEMO (www.mobilemo.com); $\quad$ MOFUSE (http://www.mofuse.com); WIRENODE (http://www.wirenode.com).

\subsubsection{Emuladores}

No es necesario conectarse vía web a través de un móvil para poder testear las páginas web ya que existe la opción de los emuladores online. Nosotros utilizaremos http://mtld.mobi/emulator.php (Figura 2), para teléfonos móviles de gama media y http://iphonetester.com, emulador del nuevo iPhone (Figura 3). 


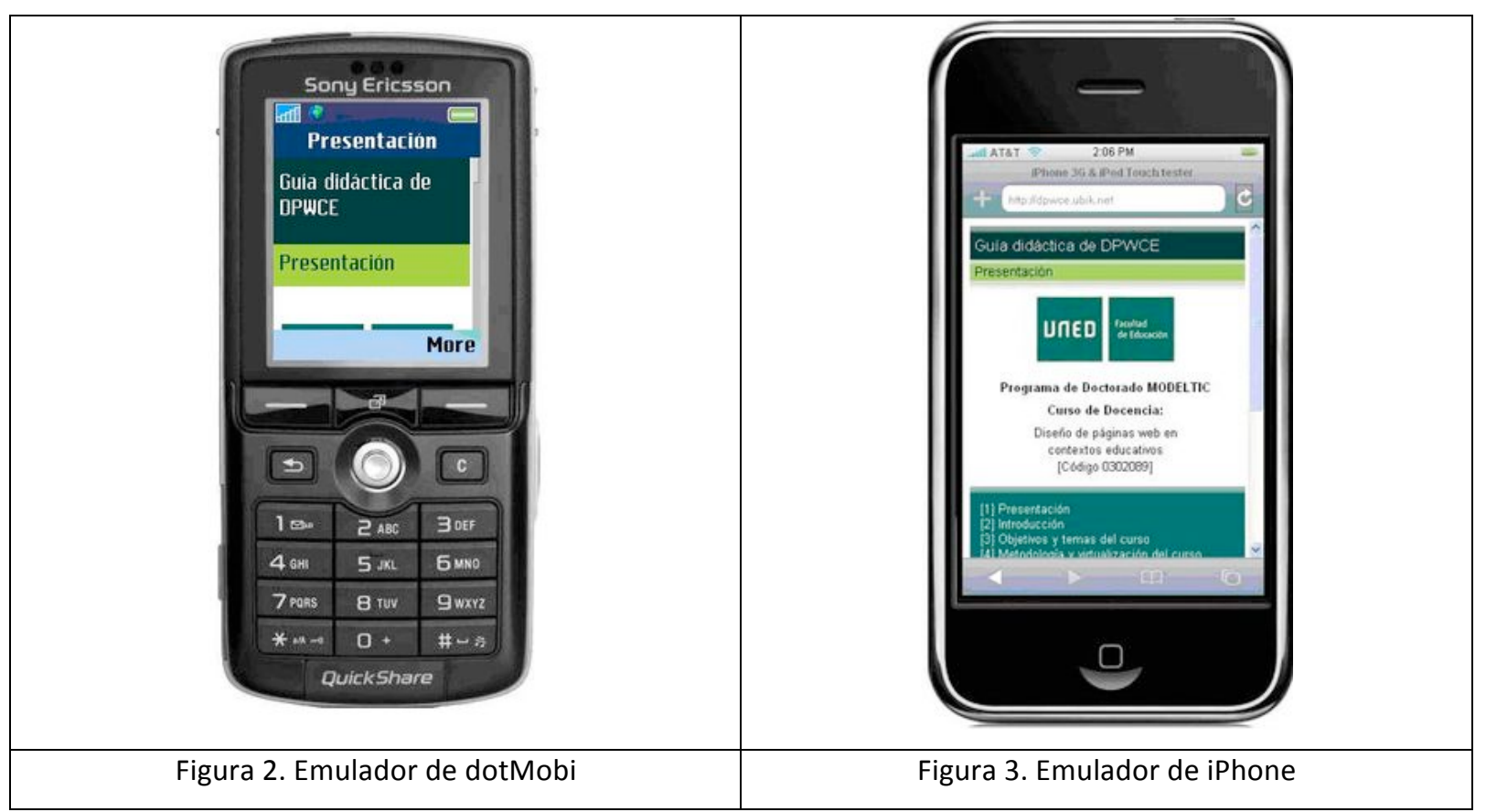

\section{OBJETIVOS DEL TRABAJO DE INVESTIGACIÓN}

A través de este trabajo teórico-práctico pretendemos:

\subsection{Establecer un marco teórico básico del teléfono móvil en educación}

2.2. Explorar las herramientas de la Web 2.0 para la creación de páginas web móviles con fines educativos

2.3.Proponer una guía metodológica para el diseño web móvil con fines educativos

\subsection{Crear la web Diseño de Páginas Web en Contextos Educativos}

\section{METOdOLOGÍA: DISEÑANDO LA WEB HTTP://DPWCE.UBIK.NET}

Para nuestra puesta en práctica, hemos optado por diseñar y crear una página web específica para dispositivos móviles. Para ello hemos seleccionado una herramienta de la Web 2.0 específica para este fin que es www.ubik.net y hemos optado por utilizar la "Guía didáctica del curso Diseño de Páginas Web en Contextos Educativos" (Cacheiro 2008), como contenido de esta página web de modo que pudiera servir, por un lado, como ejemplificación de elaboración de una página web móvil y por otro como medio útil para el acceso a esta guía de estudio a través de dispositivos móviles para futuros doctorandos. Por tanto nos encontramos ante un tipo de web informativa (en este caso de la institución del Departamento de Didáctica de la UNED) y didáctica, al tratar sobre el contenido y actividades de una determinada asignatura.

El diseño de una página web es una tarea que requiere de un adecuado planificación y, en nuestro caso, hemos optado por seguir las recomendaciones Marqués (1999 y 2007) para el diseño de páginas web educativas.

\subsection{Aspectos generales}

\subsubsection{Objetivo principal}


Diseñar una página web específica, pero no exclusivamente, para dispositivos móviles con el fin de facilitar el acceso a la información sobre la guía didáctica de la asignatura "Diseño de Páginas Web en Contextos Educativos" (DPWCE) del Departamento de Didáctica de la UNED.

\subsubsection{Destinatarios}

Todos aquellos, especialmente doctorandos, en conocer del programa de la asignatura DPWCE.

\subsubsection{Contenidos}

Los contenidos y su estructuración respetan los recogidos así en la "Guía didáctica del curso Diseño de páginas web en contextos educativos".Sin embargo, por razones técnicas, algunos apartados han tenido que ser unidos.

\subsubsection{Metodología}

Para su creación recurriremos a una herramienta Web 2.0 para la creación online de páginas web para móviles accesible en www.ubik.net (Figura 4).

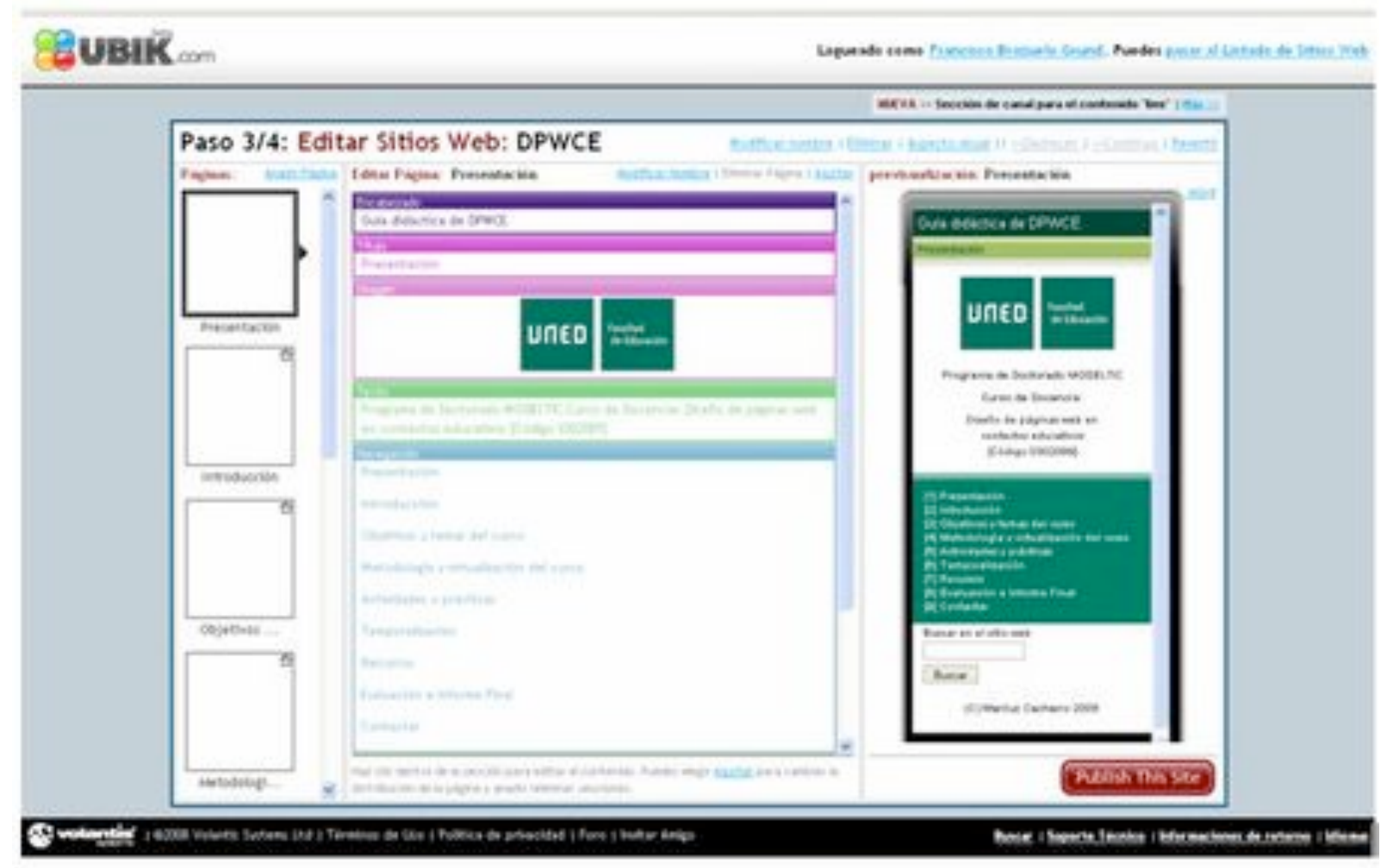

Figura 4. Página de UBIK

\subsubsection{Actividades}

Al ser una página informativa no se contemplan, pero el programa de diseño ofrece la posibilidad de crear interacciones mediante la creación de un moblog o blog para dispositivos móviles.

\subsection{Aspectos técnicos y funcionales}

Recordemos que estamos diseñando una página web para dispositivos móviles y que no es lo mismo que hacerlo para un ordenador de sobremesa. El tamaño de la pantalla y el input de los datos condicionan el diseño y el contenido. La premisa básica es la simplicidad. Aspectos 
técnicos tales como entorno visual, la navegación o las interacciones deberán ser lo más sencillas posibles para favorecer la rapidez de la conexión y la usabilidad del sitio web móvil.

\subsubsection{Contenidos}

Sobre los contenidos, se ha procurado respectar al máximo los de la guía didáctica de la asignatura "Diseño de Páginas Web en Contextos Educativos" (DPWCE), pero en algunos casos ha habido que hacer reducciones para evitar una saturación en la pantalla (recordar la otras premisa de presentar solo el contenidos relevante). Se ha empleado un tipo de letra legible y un color que contraste con el fondo de la pantalla. Solo se ha utilizado el formato de negrita para los títulos y subrayado para enlaces externos. Así mismo se han utilizado tres tamaños para la estructuración por niveles del contenido. Cada página incluye la autoría de la guía didáctica y una página para contactar.

\subsubsection{Navegación}

Dado que la navegación será en entorno de un dispositivo móvil deberá ser lo más sencilla y accesible posible. Por ello se elaborará un menú fijo en la parte inferior que contendrá la estructura de la web y que esté presente en todas las páginas web del sitio móvil, de forma que se facilite su inmediato acceso. Además se evitan los niveles en la navegación, lo que resulta incómodo cuando se trata de hacerlo por un teléfono móvil. Así mismo se evita al máximo el uso del teclado y se utilizan solo los enlaces externos necesarios. Por razones técnicas de la herramienta utilizada para crear el sitio web, éste está formado por nueve páginas. Los títulos empleados son cortos y descriptivos y sitúan al usuario en cada momento.

\subsubsection{Entorno visual}

Los colores predominantes son los verdes y el blanco que son los que utilizados en el logo de la UNED. El diseño utilizado facilita la visualización de los contenidos. No se utilizan elementos multimedia y los gráficos e imágenes se reducen a la página de Presentación y a la de Contactar. No es necesario el uso de barras de desplazamiento horizontales. De este modo la presentación resulta clara, atractiva y de fácil lectura

\subsubsection{Interacciones}

Hay un buscador en la primera página y enlaces web externos como únicos elementos de interacción.

\subsubsection{Estándares y ficheros web}

Al ser una página creada y almacena online por un servicio Web 2.0, no debemos preocuparnos por manejar o alojar ningún tipo de fichero web. Además www.ubik.net, respeta los principios de la web única y crea una página web que es válida y visualizable en cualquier tipo de dispositivo, ya sea un ordenador de sobremesa o una PDA. Además ha sido testeada en http://ready.mobi y cumple todos los estándare web recomedados desde el WC3.

\subsection{Aspectos pedagógicos}

La página web móvil creada es de carácter informativo. Los contenidos así como su extensión y organización están adaptados para este fin y son adecuados a sus destinatarios y a los objetivos que se pretenden alcanzar. En este sentido la navegación y entorno visual facilitan el acceso a la información y su comprensión evitando la sobrecarga de elementos o elementos superfluos. Las interacciones son adecuadas al carácter del sitio web y facilitan la búsqueda de información y el contacto docente. 


\subsection{Estructura del sitio web}

La web sigue la estructura del propio curso Diseño de Páginas Web en Contextos Educativos, pero adaptado al contexto móvil. Estas son sus partes: [1] Presentación;

[2] Introducción; [3] Objetivos y temas del curso; [4] Metodología y virtualización del curso; [5] Actividades y prácticas; [6] Temporalización; [7] Recursos; [8] Evaluación e informe final; [9] Contactar

Mostramos a continuación (Figura 5) el aspecto definitivo del sitio web mostrando la versión convencional (http://dpwce.ubik.net) y móvil (visualizar en http://iphonetester.com) de la página de introducción.

\section{Página de Introducción}

\section{Guiacissea 69 DPWCE}

\section{irivatcoén}

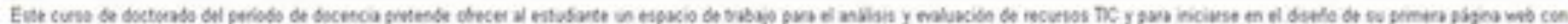

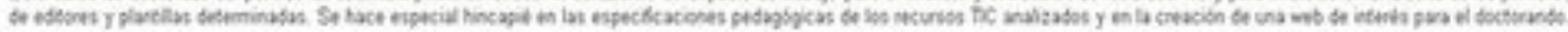

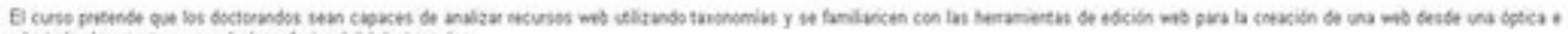

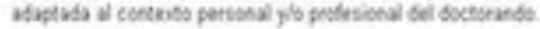

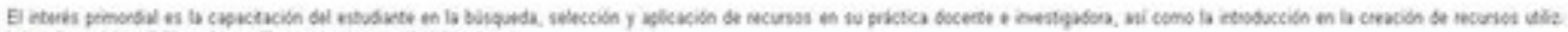

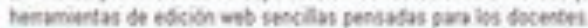

\section{Whatedscos}

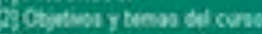

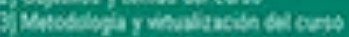

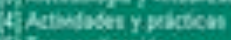

3 Tenowicisho

Fonernos

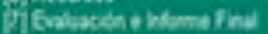

cicentury

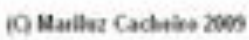




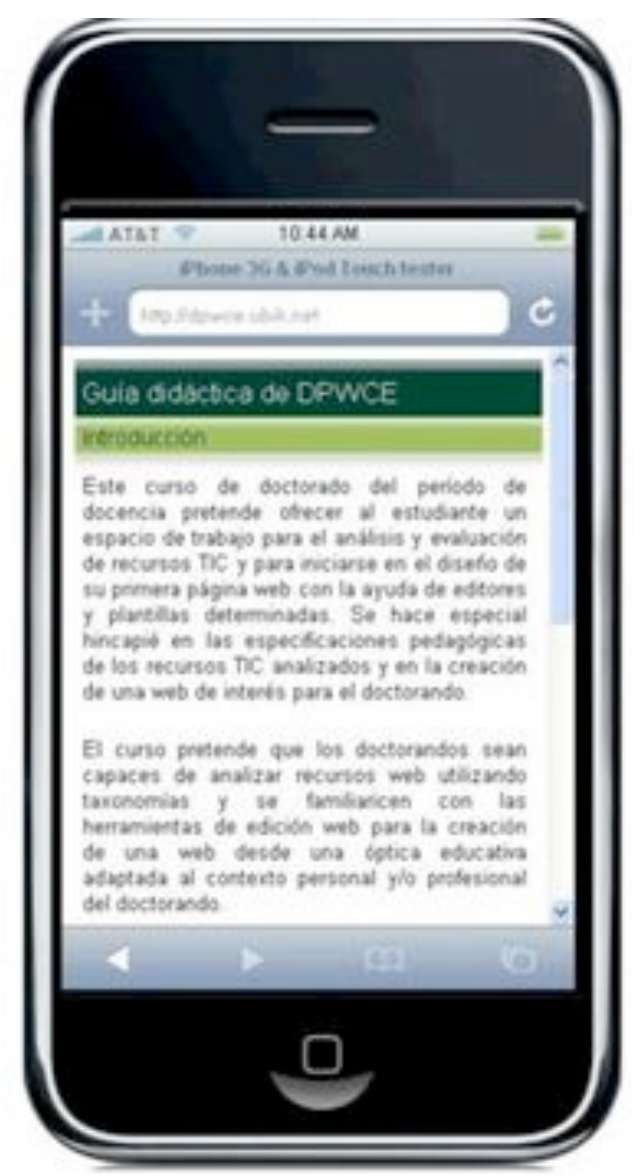

Figura 5. Versión convencional móvil de http://dpwce.ubik.net

\section{CONCLUSIONES}

La presencia de la telefonía móvil es una realidad cotidiana y una constante en la vida de nuestros jóvenes. Las nuevas generaciones, las llamadas "Nativos Digitales", es decir, las nacidas entorno a los comienzos de los años 90, han crecido rodeadas de las nuevas tecnologías, incluidas las móviles, ampliándose de modo significativo los contextos de aprendizaje hacia una total ubicuidad espacial y temporal. Cualquier escenario, real o virtual, gracias a las TIC y especialmente los dispositivos móviles, son un potencial espacio para el aprendizaje. Tal como señala García (2006): "En breve los teléfonos móviles serán capaces no sólo de acceder a los contenidos a través de Internet sino de guardar información de un cierto volumen. Los educadores lejos de responder con recelo ante otro nuevo reto deberían ir pensando ya nuevas estrategias educativas para rentabilizar la alternativa".

Por tanto no podemos cerrar las puertas a esta realidad. El mundo se hace móvil y las nuevas generaciones, interactivas, y desde las instituciones educativas debemos hacer un esfuerzo por integrar y normalizar y no por excluir estas potenciales herramientas educativas.

A través de nuestro trabajo hemos querido recorrer brevemente, en primer lugar, un campo de investigación que tiene en cuenta toda esta situación anterior y que une movilidad, aprendizaje y tecnologías móviles para dar lugar una modalidad de aprendizaje totalmente ubicua: el "Mobile Learning" (ML). 
Como ya comentábamos en nuestra fundamentación teórica, se trata de un campo relativamente reciente y aún por delimitar. Tanto es así que todavía carecemos de un marco conceptual y teórico claros sobre los que sustentar adecuadamente las investigaciones en este sentido. Pero esto no ha desanimado a los investigadores que, durante la última década, han llevado a cabo grandes avances a través de diversos estudios de carácter experimental en diversos contextos educativos hasta concluir el alto potencial pedagógico del ML. De hecho hemos visto nacer incluso una nueva didáctica determinada por las características y posibilidades de estos dispositivos tecnológicos: el "microlearning".

Convencidos del potencial del teléfono móvil como herramienta educativa, no queríamos ofrecer únicamente una perspectiva teórica sino práctica además en nuestro trabajo. Siendo conscientes del rápido crecimiento que está teniendo el acceso a Internet a través de los teléfonos móviles y en el marco de actividades y prácticas de la presente asignatura, a saber, Diseño de páginas web en contextos educativos (DpWcE), decidimos poner nuestro granito de arena en mostrar usos educativos de la telefonía móvil, proponiendo el diseño y creación de una página web específica para este medio comunicativo como tarea central para el trabajo de esa asignatura.

Y para ello contábamos con una poderosa aliada: la Web 2.0, que unida al ML daba lugar a la "Mobile Web 2.0". Haciendo uso de herramientas y aplicaciones de esta segunda generación web, creamos una página web móvil a partir de la adaptación de la Guía didáctica del curso Diseño de páginas web en contextos educativos, de modo que sirviera no solo como práctica de este alumno sino con visos a ser de utilidad para futuros doctorandos.

\section{BIBLIOGRAFIA}

Ahonen T. Y Moore, A. (2008): Bigger than TV, bigger than the internet: Understand mobile of 4 billion users, accessible en http://communities-dominate.blogs.com/brands/2009/02/biggerthan-tv-bigger-than-the-internet-understand-mobile-of-4-billion-users.html [consultado el 03/02/2009]

Banerjee, S. y Dholakia, R. (2008):"Does Location Based Advertising Work?" International Journal of Mobile Marketing, 3, 2

Cacheiro, M.L. (2008). Guía didáctica del Curso de Doctorado "Diseño de páginas web en contextos educativos", Madrid, UNED

Caudill, J. G., (2007). "The growth of m-learning and the growth of mobile computing: Parallel developments", International Review of Research in Open and Distance Learning, 8, 2

Copola, P., Lomuscio, R., Mizzaro, S y Nazzi, E. (2008). Mobile \& Web 2.0 services intergration for cultural heritage, accesible en http://km.aifb.unikalrsruhede/ews/swkm2008/coppolaetal.pdf [consultado el 05/06/2009]

Durán, M. (2009). Informe mensual N-economía: Sociedad de la Información e Innovación en el mundo, accesible en www.n-economia.com/informes.../informe mensual JUL.pdf [consultado el 15/06/2009]

García, A. (2007). Diseñar para la web móvil, accesible en www.pixelovers.com/disenar-webmovil-16029.htm [consultado el 18/07/2009] 
Georgiev, T., Georgieva, E. y Trajovski, G. (2006). Transitioning from e-Learning to m-Learning, Proceedings of the Seventh ACIS Internacional Conference on Software Engineering, 349 - 353

Kolb, L. (2008). Toys to Tools, Washington, ISTE

Kukulska-Hulme, A y Traxler, J. (2005). Mobile Learning, London, Routledged

Kukulska-Hulme, A y Traxler, J. (2006). Learning Design with Mobile and Wireless Technologies, accessible en http://kn.open.ac.uk/public/getfile.cfm?documentfileid=9882 [consultado el 05/12/2007]

Kukulska-Hulme, A. (2007). "Current Uses of Wireless and Mobile Learning", accessible en http://www.jisc.ac.uk/uploaded documents/Current\%20Uses\%20FINAL\%202005.doc

[consultado el 05/12/2007]

Marqués, P.: (1999): "La educación informal en los albores del siglo XXI", accessible en www.pangea.org/peremarques/eparalel.htm [consultado el 22/08/2009]

Marqués, P.: (2007): La Web 2.0 y sus aplicaciones didácticas, accesible en www.peremarques.net/web20.htm [consultado el 22/08/2009]

Nations, D. (2009): "What is Mobile 2.0?", en http://webtrends.about.com/od/mobileweb20/a/whatis-mobile20.htm [consultado el 18/07/2009]

O'Reilly (2005).What is Web 2.0: Design patterns and business models for the next generation of software, accessible en http://oreilly.com/web2/archive/what-is-web-20.html [consultado el 09/08/2009]

Parsons, D. y Ryu, H. (2006). A framework for assessing the quality of mobile learning accesible en http://www.masey.ac.nz/ hryu/M-learning.pdf [consultado el 10/08/2009]

Quinn, C. (2000) mLearning: Mobile, Wireless, in your Pocket Learning, accessible en http://www.linezine.com/2.1/features/cqmmwiyp.htm. [consultado el 09/08/2009]

Sharples, M., Taylor, J., y Vavoula, G. (2007). A theory of learning for the mobile age. In the Sage handbook of elearning research, London, Sage accessible en www.Isri.nottingham.ac.uk/.../Theory\%20of\%20Mobile\%20Learning.pdf [consultado el 13/03/2008]

Sharples, M., y Vavuola, G.N. (2002). KLeOS: A personal, mobile, knowledge and learning organization system, IEEE International Workshop on Mobile and Wireless Technologies in Education, Sweden accessible en www.eee.bham.ac.uk/vavoula/publications/wmte02V.pdf [consultado el 09/08/2009]

Snell, S. (2009): Mobile Web Design Trends 2009, accessible en http://www.smashingmagazine.com/2009/01/13/mobile-web-desing-trends-2009 [consultado el 15/08/2009] 
Tercer Observatorio de Tendencias Nokia (2009), accessible en www.todomovil.com/...observatorio-de tendencias...nokia.../pdf.html [consultado el 15/08/2009]

Traxler, J. (2005) Mobile Learning: It's here but what is it? accessible en http://www2.warwick.ac.uk/services/cap/resources/interactions/archive/issue25/traxler/ [consultado el 26/08/2007]

Villa, L. (2007): Como diseñar para la web móvil, accesible en http://www.alzado.org/articulo.php?id art=668 [consultado el 01/08/2009]

Wagner, E.D. (2005). "Enabling Mobile Learning" EDUCASE Quarterly Vol. 40, 3, 40-53

Winters, N. (2006) 'What is mobile learning?', In M. Sharples (ed.) Big Issues in Mobile Learning: Report of a workshop by the Kaleidoscope Network of Excellence Mobile Learning Initiative Nottingham: University of Nottingham.

Para citar este artículo:

BRAZUELO, Francisco; CACHEIRO, María Luz (2010) « DISEÑO DE PÁGINAS WEB EDUCATIVAS PARA TELÉFONOS MÓVILES » [artículo en línea]. EDUTEC, Revista Electrónica de Tecnología Educativa. Núm. 32 / Mayo 2010. [Fecha de consulta: dd/mm/aa]. http://edutec.rediris.es/revelec2/revelec32/ISSN 1135-9250. 